$\$$ Research Square
Preprints are preliminary reports that have not undergone peer review.

They should not be considered conclusive, used to inform clinical practice, or referenced by the media as validated information.

\title{
Correlation study between flash dual source CT perfusion imaging and regional lymph node metastasis of non-small cell lung cancer
}

\section{Tingting Huang}

Affiliated Hospital of Zunyi Medical University

Hui Sun

Affiliated Hospital of Zunyi Medical University

Xianli Luo

Affiliated Hospital of Zunyi Medical University

Xuemei Zhang

Affiliated Hospital of Zunyi Medical University

Kaiyuan Jin

Affiliated Hospital of Zunyi Medical University

\section{Feng Wang}

Affiliated Hospital of Zunyi Medical University

Lv Sun

Affiliated Hospital of Zunyi Medical University

Nianlan Cheng

Affiliated Hospital of Zunyi Medical University

\section{Shuo Wu}

Affiliated Hospital of Zunyi Medical University

\section{Qin Lou}

Affiliated Hospital of Zunyi Medical University

Bangguo Li ( $\square$ lbg6288@163.com )

Affiliated Hospital of Zunyi Medical University https://orcid.org/0000-0002-8226-9526

\section{Research article}

Keywords: non-small lung cancer; lymph node metastasis; computed tomography perfusion imaging; microvessel density; luminal vessels

Posted Date: May 4th, 2020

DOI: https://doi.org/10.21203/rs.2.24828/v2 
License: (c) (i) This work is licensed under a Creative Commons Attribution 4.0 International License. Read Full License

Version of Record: A version of this preprint was published at BMC Cancer on June 12th, 2020. See the published version at https://doi.org/10.1186/s12885-020-07032-8. 


\section{Abstract}

Background: To explore the correlation of flash dual source computed tomography perfusion imaging (CTPI) and regional lymph node metastasis of non-small cell lung cancer (NSCLC), and to evaluate the value of CT perfusion parameters in predicting regional lymph node metastasis of NSCLC.

Methods: 120 consecutive patients with NSCLC confirmed by postoperative histopathology were underwent flash dual source CT perfusion imaging in pre-operation. The CT perfusion parameters of NSCLC, such as blood flow (BF), blood volume (BV), mean transit time (MTT) and permeability (PMB) were obtained by the image post-processing. Then microvessel density (MVD), luminal vascular number (LVN), luminal vascular area (LVA) and luminal vascular perimeter (LVP) of NSCLC were counted by immunohistochemistry. These cases were divided into group A (patients with lymph node metastasis, 58 cases) and group B (patients without lymph node metastasis, 62 cases) according to their pathological results. The $\mathrm{CT}$ perfusion parameters and the microvessel parameters were contrastively analysed between the two groups. Receiver operating characteristic (ROC) curve was used to assess the diagnostic efficiency of CT perfusion parameters in predicting regional lymph node metastasis of NSCLC in preoperation.

Results: Group A presented significantly lower LVA, BF and higher MTT, PMB than Group B $(P<0.05)$, while $B V, L V N, L V P$ and MVD were no significant difference ( $P>0.05)$. Correlation analysis showed that BF was correlated with LVA and LVP $(P<0.05)$, while BV, MTT and PMB were not correlated with LVN, LVA and LVP $(P>0.05)$. All the perfusion parameters were not correlated with MVD. According to the ROC curve analysis, when $\mathrm{BF}<85.16 \mathrm{ml} / 100 \mathrm{ml} / \mathrm{min}$ as a cutoff point to predict regional lymph node metastasis of NSCLC, the sensitivity, specificity, accuracy, positive predictive value and negative predictive value were $60.8 \%, 81.7 \%, 71.5 \%, 75.6 \%$ and $69.5 \%$ respectively.

Conclusion: Flash dual source CT perfusion imaging can non-invasively indicate the luminal vascular structure of tumor and BF can be used as one of the important indexes in predicting regional lymph node metastasis of NSCLC in pre-operation.

\section{Background}

Lung cancer has been the leading cause of cancer death, in which non-small-cell lung accounts for more than $80 \%$ of the total [1]. So accurate staging of non-small cell lung cancer (NSCLC) is vital for the prognosis of patients. And in this process, lymph node metastasis is one of the important factors [2]. At present, CT diagnosis of NSCLC with or without regional lymph node metastasis is mainly based on the threshold of $10 \mathrm{~mm}$ short diameter of lymph node, but there are a certain false negative rate and false positive rate [3,4]. FDG-PET/CT is currently the most important noninvasive method for diagnosing lymph

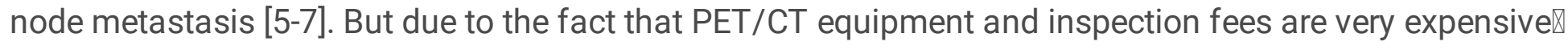
it has not been widely used, especially in China. Tumor angiogenesis is an important factor affecting tumor growth, invasion, metastasis and prognosis. It was reported that lymph node metastasis of cervical 
cancer was closely related to tumor angiogenesis [8]. Microvessel density (MVD) and luminal vascular parameters including the luminal vessels number (LVN), the luminal vessels area (LVA) and the luminal vessels perimeter (LVP) are important indicators of tumor angiogenesis. But this method belongs to invasive examination, and can not dynamically observe the angiogenesis of living tissues. Computed tomography perfusion imaging (CTPI) can reflect noninvasively the angiogenesis of tumor. It has important application value in quantitative and qualitative research of tumor [9]. Previous studies have shown that the correlation between CT perfusion parameters and luminal vascular parameters is better than MVD [9-11]. Flash dual source CT with advanced radiation reduction technology can greatly protect the health and safety of patients, so it is more suitable for CTPI research. Therefore, this study intends to introduce the luminal vascular parameters (LVN, LVA and LVP) to quantitatively analyze the microvascular structure of NSCLC and to make correlation analysis with flash dual source CT perfusion parameters in order to provide the theoretical basis in studying the CT perfusion imaging characteristics of NSCLC with regional lymph node metastasis, then to evaluate better the value of flash dual source CT perfusion parameters in predicting regional lymph node metastasis of NSCLC in pre-operation.

\section{Methods}

\section{Patients}

This study has been approved by the ethics committee of Zunyi Medical University. And each patient or the patient's family was fully informed and signed the informed consent before performing the preoperative CT examination. From January 2015 to May 2019, 120 consecutive patients with NSCLC confirmed by histopathology were enrolled in the study according to the following criteria: (i) All cases successfully underwent CT perfusion scanning preoperative and accepted surgical treatment; (ii) No treatment in pre-operation, such as chemotherapy or radiotherapy; (iii) less than 2 weeks between the time of the CT perfusion imaging and the surgery; (iv) absence of severe heart, lung or kidney insufficiency. Of these cases, 77 patients is males and 43 patients is females. The mean age was 54.35 years (range, 25-74 years). The cases were divided into group A ( patient with lymph node metastasis ) and group B (patient without lymph node metastasis) according to their pathological results.

\section{Equipment and methods}

CT perfusion scan was performed by using SOMATOM Definition Flash dual source CT scanner (Siemens, Germany) with Care dose 4D technology and X-care technology. CT scan involved two steps. First, an unenhanced CT scan was performed to determine the section of perfusion scan with following scan parameters: $100 \mathrm{kV}, 100 \mathrm{mAs}, 0.5$ pitch, $512 \times 512 \mathrm{~mm}$ matrixs and slice thickness was $8 \mathrm{~mm}$, scanned from the thoracic inlet to the bottom of the lung. Second, a perfusion CT scan in the condition of breathless which covered upper and lower poles of lung tumor (the scanning range is the upper and lower $3.5 \mathrm{~cm}$ centered at the center of lung tumor) was performed with following parameters: $80 \mathrm{kV}, 120 \mathrm{mAs}, 3$ $\mathrm{mm}$ reconstruction slice thickness (reconstruction interval $2 \mathrm{~mm}$ ) in B20f smooth Eva image reconstruction method after injecting contrast medium (iohexol) $2-4$ seconds. $50 \mathrm{ml}$ iohexol $(300 \mathrm{mgl} / \mathrm{ml})$ 
was injected via an antecubital fossa vein at a flow rate of $6 \mathrm{ml} / \mathrm{second}$, followed by $20 \mathrm{ml}$ of saline flush. In our study, CTDIvol is $₫ 60.89 \pm 8.27 \rrbracket \mathrm{mG}$ and DLP is $(536.28 \pm 45.06) \mathrm{mGy} . \mathrm{cm}$.

\section{Image post-processing}

CT perfusion imaging data of lung tumor were transmitted to workstation (Siemens Syngo Multimodality workplace) and post-processed by the volume perfusion computed tomography (VPCT) body software to obtain the perfusion parameters of NSCLC, such as blood flow (BF), blood volume (BV), mean transit time (MTT), permeability (PMB). The region of interest (ROI) of reference blood vessel was set in the thoracic aorta (if the plane of lung tumor was not present in the thoracic aorta, the arteria carotis or truncus brachiocephalicus was chosen). And the ROI of lung tumor was drawn freehand around the boundary (2-3mm from the edge of the tumor) of the tumor (avoid necrosis area, calcification areas and blood vessels) in the maximum section $\mathbb{\text { If }}$ any artifact interference, the section will is not regarded as the analysis section $囚$. Of 120 cases, 3 cases of necrosis and 1 case of calcification. Two experienced radiologists (both more than six years of experience and same level in interpreting CT perfusion imaging) were invited to do the experiments without knowing the patients' information and take the average value from them as the final data. The two experienced radiologists would be asked to calculate again if the difference between their data beyond $10 \%$.

\section{Histopathologic study}

After surgery, tumor tissue specimens were fixed in the inflated state by $10 \%$ buffered formalin, and embedded in paraffin. The tissue samples ( $3 \mu \mathrm{m}$ paraffin slice) that came from the maximum section of tumors which diagnosed to be NSCLC by hematoxylin and eosin staining were immunohistochemically stained by using the PV 2-Step method (PV-9000 2-step plusPoly-HRP Anti-Mouse/Ribbit IgG Detection System, Golden Bridge Co., Beijing, China) with CD34 (Golden Bridge Co., Beijing, China) monoclonal antibody according to the following steps: (i) removing paraffin of the tissue slices (ii) the slices were incubated in $3 \%$ nonionic hydrogen peroxide for 10 minutes and then washed with phosphate-buffered saline(PBS) three times (2 minutes each time). (iii) addition of anti-human CD34 monoclonal antibody in a 1:50 dilution for 1 hour at room temperature and then washed with PBS three times (2 minutes each time). (iv) addition of reagent 1 (Polymer Helper) for 20 minutes at room temperature, then washed with PBS three times ( 2 minutes each time). Then addition of reagent 2 (Polyperoxidase- anti-mouse/ribbit $\lg G$ ) as the method of reagent 1. (v) the slices were then stained with DAB solution and subsequently counterstained with hematoxylin. (vi) the slices were then rinsed with distilled water, dried and mounted. The tissue slices were stained with -smooth muscle actin (SMA) in the same method as CD34 staining. The control group was set up with PBS solution instead of the first antibody respectively.

\section{Quantification of histologic microvessel parameters}

Each slice was scanned at low magnification $\llbracket \times 40$ or $\times 100 \rrbracket$ to determine six "hot spot" areas where the number of microvessels was at maximum including four peripheral areas and two center areas. In each area, one field of $0.20 \mathrm{~mm}^{2}(\times 200$ magnification, $0.512 \mathrm{~mm} \times 0.383 \mathrm{~mm})$ was chosen randomly for the 
purposes of counting and measure MVD, LVN, LVP and LVA. MVD were counted in the chosen field at high magnification $(\times 200)$. Any CD34 highlighted endothelial cell or cell cluster that was apparently separate from peripheral tissues and connective tissues was counted as a single microvessel and branch construct with discrete breaks was also counted as a single microvessel. Taking the mean value of the data that came from the six fields above as the final MVD. For LVN, LVP and LVA, only microvessels with a discernible lumen and one or more complete layer(s) of -smooth muscle actin stained pericytes and smooth muscle cells (regarded as relatively mature tumor vessels) will be counted. Software (image pro plus 6.0) was used for vascular measurements. If the slices exist following situations: (i) the pathological tissue slip off; (ii) there are air bubbles; (iii) uneven staining; (iv) the background is not clean because the non-specific staining, will be excluded. Two pathologists (both more than 15 years of experience and same level in lung pathology) were invited to count each slice respectively. If the difference between the two results is more than $10 \%$, they would be asked to do it again. The mean of the two or four results is the final histologic data.

\section{Statistical analysis}

All statistical analyses were performed by using statistical software (SPSS 18.0). $P<0.05$ is considered to indicate a statistical significance. All data, including the perfusion parameters (BF, BV, MTT and PMB) and the microvessel parameters (MVD, LVN, LVP and LVA) were underwent consistency test within interobserver and intra-observer. All continuous variables were underwent normality test. Normal distribution data were expressed as mean $\pm S D$, and abnormal distribution data were represented as

median \pm interquartile range (IQR). The two-tailed Student $t$ test or Wilcoxon test was used to compare the perfusion parameters and the microvessel parameters between the group $A$ and the group $B$. Pearson or Spearman correlation analysis was used to investigate the relationships between the perfusion parameters and the microvessel parameters. Receiver operating characteristic (ROC) curve was used to assess the diagnostic efficiency of CT perfusion parameters in predicting regional lymph node metastasis of NSCLC.

\section{Results}

\section{General characteristics of patients}

Of these cases, group A includes 58 cases (33 cases of adenocarcinoma, 24 cases of squamous cell carcinoma, and 1 case of large cell undifferentiated carcinoma ) and group B includes 62 cases (32 cases of adenocarcinoma, 26 cases of squamous cell carcinoma, 2 case of large cell neuroendocrine carcinoma and 1 case of sarcomatoid carcinoma). For pN-stage, there were $62,23,35$ and 0 cases in pN0, 1, 2 and 3, respectively. For pT-stage, there were 51, 46, 23 and 0 cases in pT1, 2, 3 and 4, respectively (Table 1). In group A, the primary tumor is from $1 \mathrm{~cm}$ to $3 \mathrm{~cm}$ in length and the short diameter of lymph node was less than or equal to $10 \mathrm{~mm}$ in 11 cases (25 lymph nodes), there were 31, 20, 7 in well-differentiated, moderately differentiated and poorly differentiated, respectively. While in group $B$, the primary tumor is from $1 \mathrm{~cm}$ to $4 \mathrm{~cm}$ in length and the short diameter of lymph node was greater than 10 
$\mathrm{mm}$ in 8 cases (12 lymph nodes), there were 58, 1, 3 in well-differentiated, moderately differentiated and poorly differentiated, respectively.

\section{The microvessel parameters and CT perfusion parameters between group A and group B}

All parameters, including the microvessel parameters (MVD, LVN, LVP and LVA) and CT perfusion parameters (BF, BV, MTT and $\mathrm{PMB}$ ) were underwent consistency test within inter-observer and intraobserver. The Kappa value of the perfusion parameters within inter-observer and intra-observer is 0.86 and 0.81 respectively, and the Kappa value of the microvessel parameters within inter-observer and intraobserver is 0.85 and 0.82 respectively.

The microvessel parameters and CT perfusion parameters of group A and group B were compared. The result showed that group $A$ presented significantly lower $L V A, B F$ and higher MTT, PMB than group $B$ $(P=0.027,0.006,0.011$ and 0.048 , respectively). There were no significant difference in BV, LVN, LVP and MVD (P>0.05) (Table 2, Fig. 1, 2).

\section{Relationship of CT perfusion parameters with microvessel parameters}

Correlation analysis showed that BF was correlated with LVA and LVP $(r=0.335,0.383$, respectively; $P=$ $0.031,0.012$, respectively), while BV, MTT and PMB were not correlated with LVN, LVA and LVP $(P>0.05)$. All the perfusion parameters were not correlated with MVD ( $>0.05)$ (Table 3).

\section{The efficiency of CT perfusion parameters in diagnosing regional lymph node metastasis of NSCLC}

According to the above results, the $\mathrm{CT}$ perfusion parameter $\mathrm{BF}$, which is different between group $\mathrm{A}$ and group $B$ and correlated with the luminal vascular parameters was selected as the index to predict NSCLC with or without regional lymph node metastasis. ROC was used to test the ability of BF to diagnose regional lymph node metastasis of NSCLC. The area under ROC curves (AUC) for BF was $0.746(P<0.05)$. According to the ROC curve analysis, when $\mathrm{BF}<85.16 \mathrm{ml} / 100 \mathrm{ml} / \mathrm{min}$ as a cutoff point to predict regional lymph node metastasis of NSCLC, the sensitivity, specificity, accuracy, positive predictive value and negative predictive value were $60.8 \%, 81.7 \%, 71.5 \%, 75.6 \%$ and $69.5 \%$ respectively (Fig. 3 ).

\section{Discussion}

\section{Tumor angiogenesis and regional lymph node metastasis of NSCLC}

Tumor angiogenesis is an important factor affecting tumor growth, invasion, metastasis and prognosis [12-14]. The lymph node metastasis of tumor cells has three common ways: (i) Tumor cells directly invade the lymphatic vessels; (ii) Tumor cells directly invade into the micro vessels, and then through the incomplete basement membrane into the tumor stroma, and then enter the lymphatic vessels leading to lymph node metastasis; (iii) Tumor cells that entered into the blood circulation may also enter into the stroma of tumor or tissue with blood flow and cause lymph node metastasis. Obviously, in the process, the value of tumor angiogenesis is significant $[14,15]$. It was reported that lymph node metastasis of 
cervical cancer was closely related to tumor angiogenesis [8]. This study also showed that regional lymph node metastasis of NSCLC was related to the luminal vascular parameters, and the luminal vascular parameter LVA in group A are lower than group B, while no correlation with MVD. We believe that regional lymph node metastasis of NSCLC is more closely associated with the luminal vascular, and because of MVD including the vessels with and without lumen [16], the different proportion of them may cause the relationship between MVD and lymph node metastasis different too. Besides, the microvascular wall is mostly thin-walled and fissured, and even if some of the lumen is formed, it is also no function. Therefore, although MVD is considered to be a reliable indicator of tumor angiogenesis [17,18], luminal vascular parameters may be a better indicator for the evaluation of tumor biological behavior. But the results of this study show that there were no significant difference in LVN and LVP, we found that no matter in group A or group B, the morphology of vascular lumen was inconsistent. In group A, the lumen was mostly oval, while in group $B$, the blood vessels tended to be more round. Our analysis may be due to two reasons. First, because of the high density of tumor cells in group A, the interstitial space is small. The second reason is that the low maturity of vascular smooth muscle lead to the lumen lack of tension.

\section{The current situation of preoperative assessment of NSCLC with lymph node metastasis}

At present, CT and PET-CT are the main non-invasive methods for preoperative assessment of NSCLC with lymph node metastasis. The size of lymph nodes is the main basis of CT in judging regional lymph node metastasis of NSCLC. The short diameter of lymph node is usually $10 \mathrm{~mm}$ as the threshold for the diagnosis of lymph node metastasis, but it is easy to lead to false positive and false negative [3,4]. Moreover, the metastatic lymph nodes less than $10 \mathrm{~mm}$ in short diameter were also easily misdiagnosed in surgical operation and pathological examinations. This study showed that the short diameter of lymph node was less than or equal to $10 \mathrm{~mm}$ in 11 cases ( 25 lymph nodes) of lymph node metastasis group, while greater than $10 \mathrm{~mm}$ in 8 cases (12 lymph nodes) of non-lymph node metastasis group. This also shows that it leads easily to false positivity and false negativity, if the size of lymph nodes is used to judge whether the lymph node are metastatic or not. The lymph node greater than $10 \mathrm{~mm}$ in short diameter is not necessarily metastasis, but less than $10 \mathrm{~mm}$ may also be metastasis.

FDG-PET/CT is currently the most important noninvasive method for diagnosing lymph node metastasis. Maximal standardized uptake value (SUVmax) is a good index marker for the diagnosis of metastatic lymph nodes, but there is no consensus on the optimal threshold of SUVmax, and some non-metastatic lymph nodes can also have high uptake of 18 F-FDG. it may also lead to false positive and false negative $[19,20]$. In addition, due to the fact that PET/CT equipment and inspection fees are very expensive冈it has not been widely used, especially in China.

\section{The value of CT perfusion parameters in diagnosing regional lymph node metastasis of NSCLC in pre- operation}

CTPI can provide qualitative and quantitative hemodynamic information, and it can reflect noninvasively the angiogenesis of tumor $[9,21,22]$. It has important application value in quantitative and qualitative research of tumor. This study showed that CT perfusion parameter BF was correlated with the luminal 
vascular parameters LVA. All the perfusion parameters were not correlated with MVD. BF refers to the flow rate of blood in unit time and volume. That is to say, the larger the LVA, the larger the blood flow rate and the blood volume of per unit volume and time. This demonstrates that the dual source CT perfusion parameters can indicate the luminal vessels of tumor, but as for MVD is uncertain. This may also be associated with the microvessels including some non-functional vessels.

It was reported that CTPI plays an important role in evaluating lymph node metastasis of cancer in preoperation [23,24]. This study showed that the dual source CT perfusion parameters are related to the luminal vascular parameters of NSCLC, while the luminal vascular parameters are related to regional lymph node metastasis of NSCLC, which provides a theoretical basis for the evaluation of regional lymph node metastasis of NSCLC by CT perfusion parameters. In this study, CT perfusion parameter BF of NSCLC group with regional lymph node metastasis was lower than that of NSCLC group without regional lymph node metastasis. This showed that the dual source CT perfusion parameter BF have certain value in predicting regional lymph node metastasis of NSCLC. Our analysis may be due to following reasons. First, we found that there were more poorly differentiated tumors in group A than in group B. In group A, the lumen was mostly oval, while in group $B$, the blood vessels tended to be more round, so BF of group $A$ was lower. The second reason is that the more poorly differentiated tumors, the more obvious the destruction of blood vessels was, which was more favorable for the cancer cells to enter the interstitial tissue to have lymph node metastasis, but because of the serious destruction of blood vessels, caused the low blood flow. In addition, the immature blood vessels in poorly differentiated tumors are relatively more and disordered, which also affects the blood flow rate of the tissues to a certain extent, and the proliferation speed of poorly differentiated tumor cells is fast, and the situation of tissue ischemia and hypoxia is more. In this study, BV, MTT and PS there are no correlation with microvascular parameters. BV there is no difference in between group $A$ and group $B$, therefore, we speculated that blood volume can only reflect the total amount of blood contained in the lesion, but its value in reflecting its biological behavior is limited. PS is not only related to the integrity of endothelial cells, but also to the pressure balance between plasma and tissue fluid. Poorly differentiated tumors have relatively severe damage to tumor blood vessels, which may increase the permeability of blood vessels. However, the rapid proliferation of poorly differentiated tumor cells will leads to the increase of tissue fluid pressure. Therefore, we believe that PS are uncertain in the evaluation of lymph node metastasis. MTT there is difference in between group $A$ and group $B$, but there are no correlation with microvascular parameters, therefore, we have no reliable theoretical basis for predicting regional lymph node metastasis of NSCLC by MTT. MTT may be a potential indicator, but we need to deepen our understanding of tumor blood vessels to confirm this conclusion.

ROC curve was used to evaluate the value of the dual source CT perfusion parameter BF in predicting regional lymph node metastasis of NSCLC. The result showed that BF was valuable in predicting regional lymph node metastasis of NSCLC in pre-operation. And the possibility of NSCLC with regional lymph node metastasis may be suggested when $\mathrm{BF}<85.16 \mathrm{ml} / 100 \mathrm{ml} / \mathrm{min}$. For these patients, the regional lymph node dissection should be performed more carefully, systematically and extensively to avoid missing the small metastatic lymph nodes. 


\section{Conclusion}

This study showed that the luminal vascular parameters were the important indexes for the evaluation of tumor angiogenesis and were related to the regional lymph node metastasis of NSCLC. Flash dual source CT perfusion imaging can non-invasively indicate the luminal vascular structure of tumor and BF can be used as one of the important indexes in predicting regional lymph node metastasis of NSCLC in preoperation. So the dual source CT perfusion imaging can be an effective supplement of traditional morphological image in diagnosing regional lymph node metastasis of NSCLC.

Small sample size, a scanner from a single vendor and single-center study are the main limitations of this study. Therefore, further work is to expand the sample size and conduct multi-center research to improve the value of the CT perfusion parameters in predicting regional lymph node metastasis of NSCLC.

\section{Abbreviations}

AUC: area under the curve; BF: blood flow; BV: blood volume: CT: computed tomography; CTDIvol: volume computed tomography dose index; CTPI: computed tomography perfusion imaging; DLP: dose length product; LVA: luminal vascular area; LVN: luminal vascular number; LVP: luminal vascular perimeter; MTT: mean transit time; MVD: microvessel density; NSCLC: non-small cell lung cancer; PMB: permeability; ROC: receiver operating characteristic; ROI: region of interest; SMA: smooth muscle action; SUVmax: maximal standardized uptake value; VPCT: volume perfusion computed tomography

\section{Declarations}

\section{Ethics approval and consent to participate}

This study has been approved by the ethics committee of Zunyi Medical University. Each patient or the patient's family was fully informed and signed the informed consent before performing the pre-operative CT examination.

\section{Consent for publication}

Not applicable.

\section{Availability of data and materials}

All data generated or analyzed during this study are included in this article and its supplementary information files.

\section{Competing interests}

The authors declare that they have no competing interests.

\section{Funding}


This study is supported by a grant from National Natural Science Foundation of China (No.81460265). The funding agency played no role in the design and implementation of the study and in writing the manuscript.

\section{Authors' contributions}

HTT participated in the design of the study $₫$ carried out the most studies (CT perfusion imaging , image post-processing, image analysis, statistical analysis and so on) and drafted the manuscript. SH and LXL carried out the CT perfusion imaging, image post-processing and image analysis. ZXM, JKY, WF and SL participated in the CT perfusion imaging, image post-processing. CLN, WS and LQ participated in the data collection and arrangement. LBG conceived, designed and data analysis. All authors have read and approved the final manuscript.

\section{Acknowledgments}

We would like to thank professor Li Hong for her assistance in statistical analysis.

\section{References}

1. Oser MG, Niederst MJ, Sequist LV, et al. Transformation from non-small-cell lung cancer to small-cell lung cancer: molecular drivers and cells of origin [J]. Lancet Oncol, 2015, 16(4): e165-e172.

2. Rami-Porta R, Bolejack V, Giroux DJ, et al. The IASLC lung cancer staging project: the new database to inform the eighth edition of the TNM classification of lung cancer [J].J Thorac Oncol. 2014, 9(11): 1618-1624.

3. Xia $\mathrm{Y}$, Zhang $\mathrm{B}$, Zhang $\mathrm{H}$, et al. Evaluation of lymph node metastasis in lung cancer: who is the chief justice [J]? J Thorac Dis, 2015, 7(4):S231- S237.

4. Kudo S, Imai K, Ishiyama K, et al. New CT criteria for nodal staging in non-small cell lung cancer [J]. Clin Imaging, 2014, 38(4): 448-453.

5. Sauter AW, Spira D, Schulze M, et al. Correlation between $\left[{ }^{18} \mathrm{~F}\right] \mathrm{FDG}$ PET/CT and volume perfusion CT in primary tumours and regional lymph nodes of non-small-cell lung cancer [J]. Eur $\mathrm{J}$ Nucl Med Mol Imaging, 2013, 40(5): 677-684.

6. Shigemoto Y, Suga K, Matsunaga N. F-18-FDG-avid lymph node metastasis along preferential lymphatic drainage pathways from the tumor-bearing lung lobe on F-18-FDG PET/CT in patients with non-small-cell lung cancer [J]. Ann Nucl Med, 2016, 30(4): 287-297.

7. Wu Y, Li P, Zhang H, et al. Diagnostic value of fluorine 18 fluorodeoxyglucose positron emission tomography/computed tomography for the detection of metastases in non-small-cell lung cancer patients [J]. Int J Cancer, 2013, 132(2): E37-E47.

8. Sun $\mathrm{H}$, Tang $\mathrm{F}$, Zhou S, et al. Association between vascular endothelial growth factor expression and lymph node metastasis in cervical cancer: A meta-analysis [J]. J Obstet Gynaecol Res, 2016,42(10):1310-1316. 
9. Wang M, Li BG, Sun H, et al. Correlation study between dual source CT perfusion imaging and the microvascular composition of solitary pulmonary nodules [J]. Lung Cancer, 2019, 130(4): 115-120.

10. Kan Z, Phongkitkarun S, Kobayashi S, et al. Functional CT for quantifying tumor perfusion in antiangiogenic therapy in a rat model [J]. Radiology, 2005, 237(1): 151-158.

11. Xiong Z, Liu JK, Hu CP, et al. Role of immature microvessels in assessing the relationship between CT perfusion characteristics and differentiation grade in lung cancer [J]. Arch Med Res, 2010, 41(8):611617.

12. JaysonGC, KerbelR, EllisLM, eta1. Antiangiogenic therapy in oncology: Current status and future directions [J]. Lancet, 2016, 388(10043):518-529.

13. Ziyad S, Iruela-Arispe. Molecular mechanisms of tumor angiogenesis [J]. MLGenes Cancer, 2011, 2(12):1085-1096.

14. Che $X$, Hokita $S$, Natsugoe $S$, et al. Tumor angiogenesis related to growth pattern and lymph node metastasis in early gastric cancer [J]. Chin Med J (Engl), 1998, 111(12): 1090-1093.

15. Bielenberg DR, Zetter BR. The Contribution of Angiogenesis to the Process of Metastasis [J].Cancer J, 2015, 21(4): 267-273.

16. Boruah D, Bhatia JK, Rai A, et al. Correlation of microvessel parameters in invasive ductal carcinoma of the breast and fibroadenomas: a morphometric study [J]. Ann Diagn Pathol, 2016, 25(1): $72-78$.

17. Wang X, Li K, Sun H, et al. Tumoral angiogenesis in both adrenal adenomas and nonadenomas: a promising computed tomography biomarker for diagnosis [J]. Onco Targets Ther, 2016, 9(1): 18231830.

18. El Gehani K, Al-Kikhia L, Mansuri N, et al. Angiogenesis in urinary bladder carcinoma as defined by microvessel density (MVD) after immunohistochemical staining for Factor VIII and CD31 [J]. Libyan J Med, 2011, 6(1): 1-6.

19. Kuo WH, Wu YC, Wu CY, et al. Node/aorta and node/liver SUV ratios from (18)F-FDG PET/CT may improve the detection of occult regional lymph node metastases in patients with non-small cell lung carcinoma [J]. Acad Radial, 2012, 19(6): 685-692.

20. Suzawa N, Ito M, Qiao S, et al. Assessment of factors influencing FDG uptake in non-small cell lung cancer on PET/CT by investigating histological differences in expression of glucose transporters 1 and 3 and tumour size [J]. Lung Cancer, 2011, 72(2): 191-198.

21. Kim SH, Kamaya A, Willmann JK. CT perfusion of the liver: Principles and applications in oncology [J]. Radiology, 2014, 272 (2): 322-344区

22. Gareia-Figueiras R, Goh VJ, Padhani AR, et a1. CT perfusion in oncologic imaging: A useful tool [J]? AJR Am J Roentgenol, 2013, 200(1): 8-19.

23. Hayano K, Shuto K, Koda K, et al. Quantitative measurement of blood flow using perfusion CT for assessing clinicopathologic features and prognosis in patients with rectal cancer [J]. Dis Colon Rectum. 2009, 52(9):1624-1629. 
24. Zhou H, Xiong Z, Liu JK, et al. Low tumor blood flow assessed with perfusion CT correlates with lymphatic involvement in patients with stage T1b non-small cell lung cancer [J]. Thoracic Cancer, 2013, 4(2): 131-137.

\section{Tables}

Table 1 General characteristics of patients

\begin{tabular}{|c|c|}
\hline Characteristics & No. (\%) \\
\hline \multicolumn{2}{|l|}{ Location of the primary lesion } \\
\hline Right upper lobe & $28(23.33)$ \\
\hline Right middle lobe & $5(4.17)$ \\
\hline Right lower lobe & $24(20.00)$ \\
\hline Left upper lobe & $27(22.50)$ \\
\hline Left lower lobe & $36(30.00)$ \\
\hline \multicolumn{2}{|l|}{ Histology } \\
\hline Adenocarcinoma & $66(55.00)$ \\
\hline Squamous carcinoma & $50(41.67)$ \\
\hline Large cell neuroendocrine carcinoma & $2(1.67)$ \\
\hline Large cell undifferentiated carcinoma & $1(0.83)$ \\
\hline Sarcomatoid carcinoma & $1(0.83)$ \\
\hline \multicolumn{2}{|l|}{ Differentiation degree } \\
\hline Well-differentiated & $89(74.17)$ \\
\hline Moderately differentiated & $21(17.50)$ \\
\hline Poorly differentiated & $10(8.33)$ \\
\hline \multicolumn{2}{|l|}{ Lymph node metastasis } \\
\hline pNO & $62(51.67)$ \\
\hline pN1 & $23(19.17)$ \\
\hline $\mathrm{pN} 2$ & $35(29.16)$ \\
\hline pN3 & 0 \\
\hline \multicolumn{2}{|l|}{ T staging } \\
\hline pT1 & $51(42.50)$ \\
\hline pT2 & $46(38.33)$ \\
\hline pT3 & $23(19.17)$ \\
\hline pT4 & 0 \\
\hline
\end{tabular}

Table 2 Difference of parameters between group A and group B 


\begin{tabular}{|c|c|c|c|c|}
\hline parameters & group A & group B & $\bar{Z}$ & $\bar{P}$ \\
\hline $\mathrm{BF} \square \mathrm{ml} / 100 \mathrm{ml} / \mathrm{min} \square$ & $66.13 \pm 30.15$ & $115.57 \pm 80.21$ & -2.710 & 0.006 \\
\hline $\mathrm{BV} \square \mathrm{ml} / 100 \mathrm{ml} \square$ & $8.58 \pm 4.31$ & $9.21 \pm 3.54$ & -0.728 & 0.467 \\
\hline $\mathrm{MTT} \square \mathrm{s} \square$ & $15.85 \pm 15.92$ & $6.10 \pm 2.57$ & -2.667 & 0.011 \\
\hline $\mathrm{PMB} \square \mathrm{ml} / 100 \mathrm{ml} / \mathrm{min} \square$ & $21.18 \pm 29.87$ & $20.59 \pm 7.91$ & -1.959 & 0.048 \\
\hline MVD $\llbracket$ strip/field $\square$ & $67.21 \pm 46.69$ & $70.85 \pm 50.14$ & -0.447 & 0.637 \\
\hline LVN₫strip/fieldם & $6.70 \pm 3.10$ & $8.31 \pm 3.87$ & -1.268 & 0.218 \\
\hline LVA $\square \mu \mathrm{m}^{2} /$ field $\square$ & $4617.65 \pm 1435.67$ & $6541.37 \pm 3235.76$ & -2.310 & 0.027 \\
\hline LVP $\square$ m/field $\square$ & $718.71 \pm 216.75$ & $942.09 \pm 418.39$ & -1.951 & 0.052 \\
\hline
\end{tabular}

Abbreviations:

$\mathrm{BF}=$ blood flow; $\mathrm{BV}=$ blood volume; $\mathrm{LVA}=$ luminal vascular area; $\mathrm{LVN}=$ luminal vascular number; $\mathrm{LVP}=$ luminal vascular perimeter; $\mathrm{MTT}=$ mean transit time; $\mathrm{MVD}=$ microvessel density; $\mathrm{PMB}=$ permeability

Table 3 Relationship of CT perfusion parameters with microvessel parameters

\begin{tabular}{|c|c|c|c|c|c|c|c|c|}
\hline \multirow[t]{2}{*}{ parameters } & \multicolumn{2}{|r|}{$\mathrm{BF}$} & \multicolumn{2}{|l|}{ BV } & TTT & PMB & \multirow[b]{2}{*}{$r$} & \multirow[b]{2}{*}{$\bar{P}$} \\
\hline & $r$ & $\bar{P}$ & $r$ & $P$ & $r$ & $P$ & & \\
\hline MVD & 0.151 & 0.371 & -0.126 & 0.431 & -0.261 & 0.110 & 0.001 & 0.998 \\
\hline LVN & 0.229 & 0.140 & 0.071 & 0.650 & -0.051 & 0.761 & 0.052 & 0.750 \\
\hline LVA & 0.335 & 0.031 & 0.160 & 0.313 & -0.216 & 0.169 & 0.163 & 0.296 \\
\hline LVP & 0.383 & 0.012 & 0.259 & 0.091 & -0.138 & 0.379 & 0.070 & 0.679 \\
\hline
\end{tabular}

Abbreviations: $\mathrm{BF}=$ blood flow; $\mathrm{BV}=$ blood volume; $\mathrm{LVA}=$ luminal vascular area; $\mathrm{LVN}=$ luminal vascular number; LVP=luminal vascular perimeter; $\mathrm{MTT}=$ mean transit time; $\mathrm{MVD}=$ microvessel density; $\mathrm{PMB}=$ permeability

\section{Figures}

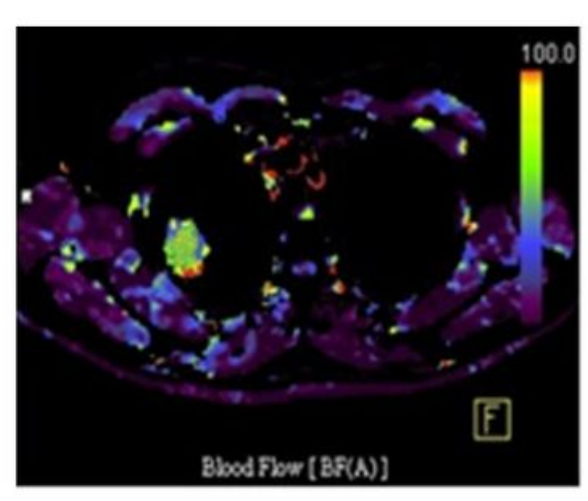

1a

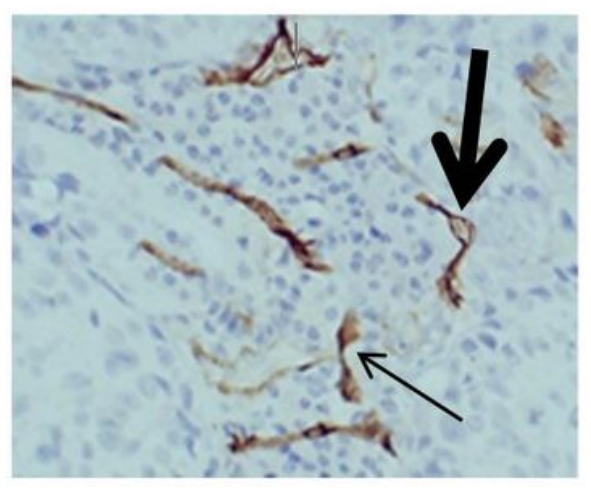

$1 \mathrm{~b}$

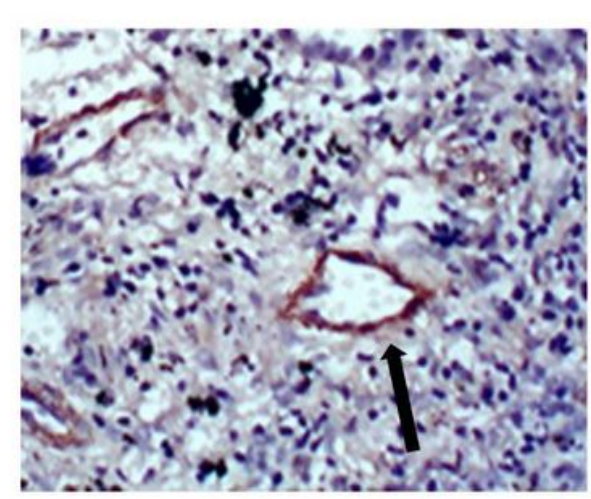

$1 \mathrm{c}$

\section{Figure 1}


A 52-year-old man with squamous cell carcinoma at the upper lobe of right lung with right hilar lymph node metastasis. (a) Functional map of perfusion showed that blood flow value was low (48.36 $\mathrm{ml} / 100 \mathrm{ml} / \mathrm{min}$ ). (b) CD34 staining showed microvessels with luminal vessels (thick arrow) and without luminal vessels (thin arrow) and the latter accounted for the main part ( $\times 200)$. (c) SMA staining showed fewer microvessels covered with completed layers of smooth muscle cells (arrows) $(\times 200)$

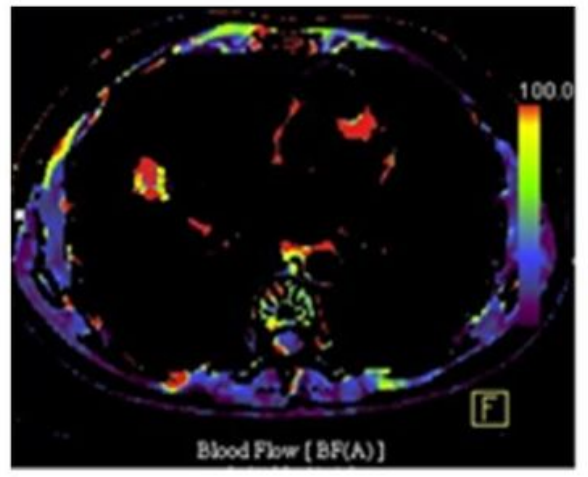

$2 \mathrm{a}$

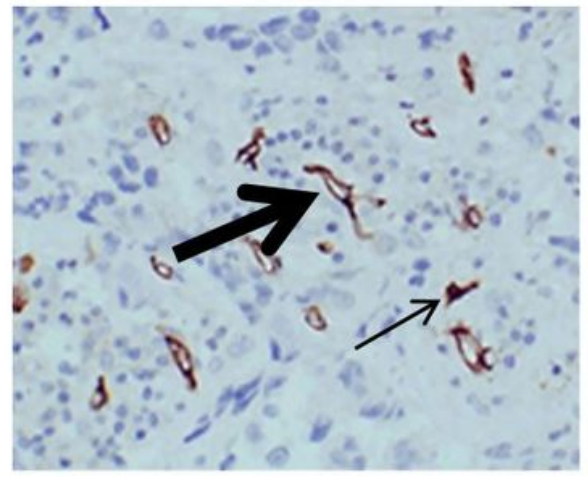

$2 b$

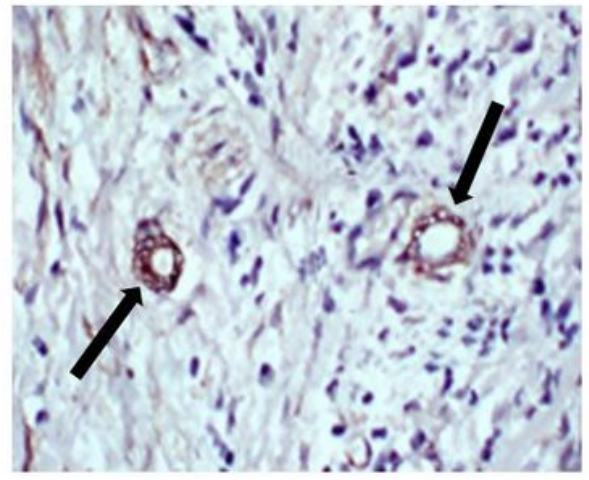

$2 \mathrm{c}$

Figure 2

A 65-year-old man with adenocarcinoma at the middle lobe of right lung without regional lymph node metastasis. (a) Functional maps of perfusion show that blood flow value was high (105.23 $\mathrm{ml} / 100 \mathrm{ml} / \mathrm{min}$ ). (b) CD34 staining showed microvessels with luminal vessels (thick arrow) and without luminal vessels (thin arrow) and the former accounted for the main part $(\times 200)$. (c) SMA staining showed more microvessels covered with completed layers of smooth muscle cells (arrows) $(\times 200)$ 
ROC Curve

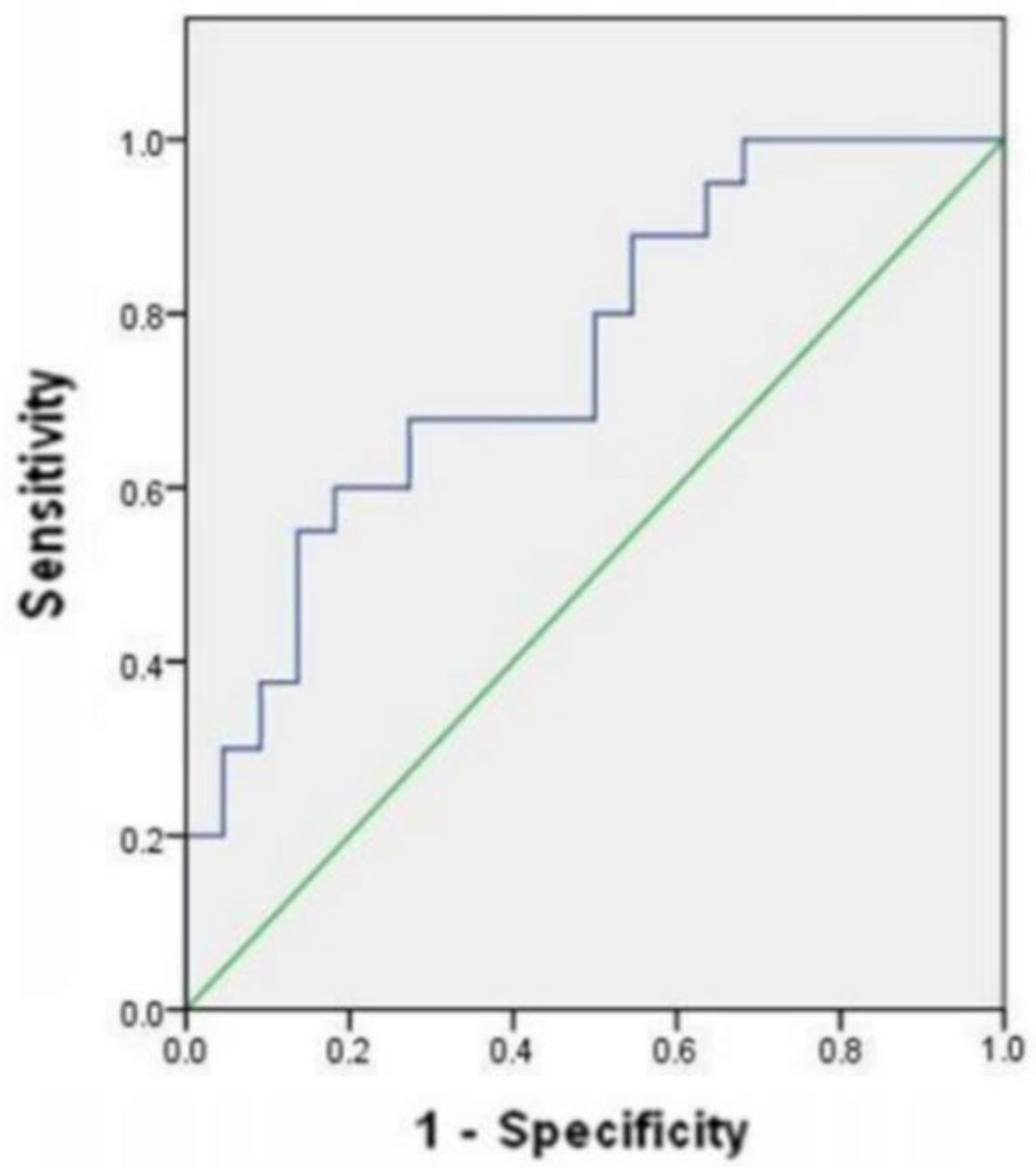

Figure 3

ROC curve of BF in predicting regional lymph node metastasis of NSCLC 